\title{
métodos para la determinación de ácido silícico con anhídrico acético, y calcio y óxido de magnesio con complesona III en conglomerantes hidráulicos
}

\section{J. F A N J U L}

\section{DETERMINACION DEL ACIDO SILICICO POR DISGREGACION CON ANHIDRIDO ACETICO}

\section{Principio}

El silicato se disgrega con anhídrido acético. Se añade agua regia para formar los correspondientes cloruros. El agua regia oxida las trazas eventuales existentes de iones ferrosos. El ácido silícico impuro con la parte insoluble y las trazas de los sesquióxidos se calcinan en el horno de mufla a $1.000^{\circ} \mathrm{C}$.

El ácido silícico puro se determina mediante su volatilización con ácido fluorhídrico.

\section{Reacción}

$$
\begin{aligned}
& \mathrm{SiO}_{2}+4 \mathrm{HF}=\mathrm{SiF}_{4} \nearrow+2 \mathrm{H}_{2} \mathrm{O} \\
& \mathrm{R}_{2} \mathrm{O}_{3}+6 \mathrm{HF}=2 \mathrm{RF}_{3}+3 \mathrm{H}_{2} \mathrm{O} \\
& 2 \mathrm{RF}_{3}+3 \mathrm{H}_{2} \mathrm{SO}_{4}=\mathrm{R}_{2}\left(\mathrm{SO}_{4}\right)_{3}+6 \mathrm{HF} \nearrow \\
& \mathrm{R}_{2}\left(\mathrm{SO}_{4}\right)_{3}+\mathrm{Q}_{\left(1.000^{\circ} \mathrm{Cl}\right.}=\mathrm{R}_{2} \mathrm{O}_{3}+3 \mathrm{SO}_{3} \nearrow
\end{aligned}
$$

\section{Causas de error}

Para obtener resultados exactos se debe seguir exactamente el método operatorio prescrito.

Si el residuo del ataque con ácido fluorhídrico no se calcina a $1.000^{\circ} \mathrm{C}$, las trazas de sesquióxidos que se hallan presentes en el ácido silícico retienen un considerable volumen de sulfato. Por esto puede originarse un grave error en el cálculo del ácido silícico.

\section{Descripción del método}

Se toma $1 \mathrm{~g}$ de muestra de cemento y se pone en un vaso de vidrio de $250 \mathrm{ml}$ (forma alta). Se añaden $10 \mathrm{ml}$ de anhídrido acético y $10 \mathrm{ml}$ de agua destilada. Se calienta el conjunto, aunque no con demasiada intensidad, hasta que se complete la reacción (solución de color rosáceo). Se añade lentamente agua regia hasta que la solución toma un color amarillo claro. Se añaden $20 \mathrm{ml}$ de agua destilada y se calienta unos 5 a 10 minutos, una vez más, no con demasiada intensidad.

El conjunto se hace pasar por el filtro (banda blanca $11 \mathrm{~cm} \varnothing$ ). El residuo se lava dos veces con ácido clorhídrico al $5 \%$ y 20 veces con agua caliente destilada. El filtro con el silicato se calienta lentamente en un crisol de platino hasta su combustión total; a continuación se trata durante 45 minutos a $1.000^{\circ} \mathrm{C}$ en el horno de mufla, se enfría durante 15 minutos en un desecador y se pesa.

Al contenido pesado del crisol de platino se añaden algunas gotas de agua, así como $5 \mathrm{ml}$ de ácido fluorhídrico y 3 gotas de ácido sulfúrico concentrado. Se deja humear sobre el 
baño de arena, se pone el crisol en el horno a $1.100^{\circ} \mathrm{C}$ (15 minutos), a continuación se hace enfriar durante 15 minutos en un desecador y se pesa.

La diferencia entre el peso del crisol de platino antes y después del tratamiento con ácido fluorhídrico proporciona el ácido silícico puro.

La diferencia entre el peso del crisol de platino vacío y el peso que tiene después del tratamiento porporciona la cantidad de sesquióxidos y producto insoluble en el ácido silícico.

\section{Reactivos empleados}

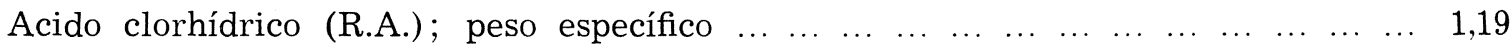

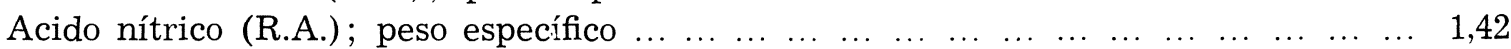

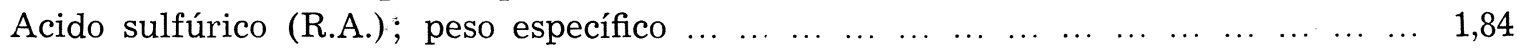

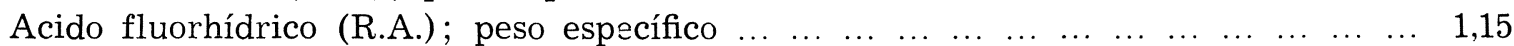

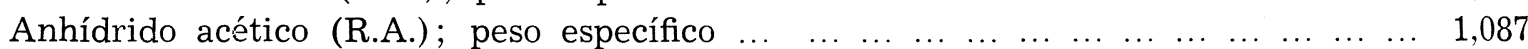

N B.

\section{Sesquióxidos}

Como método para el análisis de los sesquióxidos se puede emplear el de F. Guye, con la diferencia de que hay que añadir $2 \mathrm{~g}$ de cloruro amónico al producto filtrado del ácido silícico.

\section{DETERMINACION COMPLEXOMETRICA DE LOS OXIDOS DE CALCIO Y DE MAGNESIO}

\section{Principios complexométricos}

Para la formación del complejo se emplea la sal disódica dihidratada del ácido etilenodiaminotetracético, la llamada complexona III (E.D.T.A.), simbolizada por $\mathrm{Na}_{2} \mathrm{H}_{2} \mathrm{Y} \cdot 2 \mathrm{H}_{2} \mathrm{O}$. La reacción $\mathrm{Na}_{2} \mathrm{Y}^{2-}$ proporciona el complejo con el catión $\mathrm{M}^{2+}$ :

$$
\begin{gathered}
\mathrm{Na}_{2} \mathrm{H}_{2} \mathrm{Y} \cdot 2 \mathrm{H}_{2} \mathrm{O}+\mathrm{M}^{2+}+\mathrm{nH}_{2} \mathrm{O}=\mathrm{YNa}_{2} \mathrm{M} \cdot \mathrm{nH}_{2} \mathrm{O}+2 \mathrm{H}_{3} \mathrm{O}^{+} \\
\mathrm{M}^{2+}+\mathrm{H}_{2} \mathrm{Y}^{2-} \longleftrightarrow \mathrm{MY}^{2-}+2 \mathrm{H}^{+}
\end{gathered}
$$

Si aumenta el pH, aumenta asimismo la formación de $\mathrm{H}_{2} \mathrm{Y}^{2-}$ por disociación; en consecuencia, se desplaza el equilibro $\mathrm{MY}^{2-} \Longleftrightarrow \mathrm{M}^{2+}+\mathrm{H}_{2} \mathrm{Y}^{2-}$ hacia la izquierda. Los complejos son tanto más estables cuanto más básico es el medio.

Con $\mathrm{pH}$ 10-11 los complejos $\mathrm{YNa}_{2} \mathrm{Ca} \cdot \mathrm{nH}_{2} \mathrm{O}$ e $\mathrm{YNa}_{2} \mathrm{Mg} \cdot \mathrm{nH}_{2} \mathrm{O}$ son estables; el calcio y el magnesio se pueden valorar volumétricamente.

Con $\mathrm{pH} 13$ el hidróxido de magnesio se precipita; la valoración con E.D.T.A. proporciona sólo la cantidad de calcio.

La reacción que se desarrolla durante la valoración entre el indicador metálico y la complexona puede representarse mediante la siguiente expresión:

$$
\begin{aligned}
& 2 \text { Ind. }^{-}+\mathrm{M}^{2+}=\text { (Ind.) }{ }_{2} \mathrm{M} \\
& \text { incoloro color } \mathrm{A} \\
& \begin{array}{cc}
\mathrm{M}^{2+}+\mathrm{H}_{2} \mathrm{Y}^{2-} & =\mathrm{MY}^{2-}+2 \mathrm{H}^{+} \\
\text {incoloro } & \text { color } \mathrm{B}
\end{array}
\end{aligned}
$$

Si el complejo metal-indicador es más débil o menos consistente que el complejo metalE.D.T.A., el primer equilibrio se desplaza hacia la izquierda; y el segundo, hacia la derecha. Si el complejo metal-indicador ha desaparecido obtenemos un cambio repentino del color A o del color B; en este momento ha concluido la valoración.

\section{Causas de error}

La valoración debe hacerse rápidamente para impedir la formación de carbonato cálcico. El indicador sólo debe añadirse cuando faltan todavía 1-2 $\mathrm{ml}$ del complejo para terminar la valoración. 
El volumen del indicador debe ser muy pequeño. La solución debe agitarse bien cuando se añaden los reactivos necesarios para la valoración.

\section{Determinación del óxido de calcio}

La disgregación del conglomerante tiene lugar con ácido clorhídrico o anhídrido acético. Los sesquióxidos se precipitan con amoníaco. Los productos filtrados de los sesquióxidos se ponen en un matraz aforado de $500 \mathrm{ml}$. Para el análisis se toman $100 \mathrm{ml}$, que se diluyen en un vaso de vidrio de $600 \mathrm{ml}$ con $200 \mathrm{ml}$ de agua destilada.

Se añaden $3 \mathrm{~g}$ de hidróxido sódico en lentejas o $25 \mathrm{ml}$ de solución de hidróxido sódico $4 \mathrm{~N}$, y lo antes posible se incorpora una cantidad previamente establecida de complexona III $0,1 \mathrm{M}$, que es algo menor que la que se necesita efectivamente. A continuación se añade una pequeña cantidad de indicador puro HHSNN, y se continúa con la valoración hasta que el color cambia de rojo a azul.

Conforme con la reacción estequiométrica, $1 \mathrm{~mol}$ de complexona III es equivalente a 1 mol de óxido de calcio; por ello $1 \mathrm{ml}$ de complexona III 0,1 M corresponde a 5,608 $\mathrm{mg}$ de óxido de calcio.

El número de $\mathrm{ml}$ multiplicado por 2,804 proporciona el contenido de óxido de calcio en la muestra ensayada, en \% (con un peso de $1 \mathrm{~g}$ ).

\section{Reactivos empleados}

Hidróxido sódico (R.A.).

Solución de complexona III: $37,225 \mathrm{~g}$ de sal seca a $80^{\circ} \mathrm{C}$ en 1 litro, proporciona una solución de 0,1 M. Esta solución no debe ya valorarse.

Indicador HHSNN (R.A.).

\section{Determinación del óxido de magnesio}

Para determinar el magnesio se valora la suma de calcio y magnesio. El contenido de magnesio resulta de la diferencia entre el volumen de solución de complejo, que por una parte se consume para el calcio y por otra para la suma de calcio y magnesio. El indicador metálico que se emplea para esta última valoración es el negro de eriocromo $\mathrm{T}$. Las trazas de sales de manganeso provocan en la valoración del magnesio graves errores. Por esta razón se añade cloruro de hidroxilamina. Este actúa reduciendo el manganeso trivalente y confiere al indicador una mayor estabilidad.

Para obtener una tonalidad más clara del indicador, se debe añadir complexonato magnésico. La valoración se hace a $\mathrm{pH} 10$. Para obtener este $\mathrm{pH}$ se emplea hidróxido amónico (R.A.) en presencia de sales de amonio.

La cantidad utilizada en el proceso analítico suele ser suficiente. Impide la precipitación del magnesio en forma de hidróxido.

\section{Reacción}

Las reacciones de esta valoración corresponden a las de la valoración del calcio.

\section{Causas de error}

La valoración debe realizarse exactamente según el método prescrito para eliminar errores graves.

El indicador sólo debe añadirse al final de la valoración para obtener un punto claro de viraje.

Demasiada hidroxilamina y complexonato de magnesio proporcionan un punto final lento. 


\section{Descripción del método}

Del matraz aforado, que contiene los productos filtrados de los sesquióxidos, se toman, con una pipeta de precisión, $100 \mathrm{ml}$, que se diluyen en un vaso de vidrio de $600 \mathrm{ml}$ con $200 \mathrm{ml}$ de agua destilada.

Se añaden $3 \mathrm{ml}$ de cloruro de hidroxilamina al 2,5\%, a continuación $3 \mathrm{ml}$ de complexonato de magnesio al $3 \%$ y finalmente, $30 \mathrm{ml}$ de hidróxido amónico (R.A.). Se vierte la cantidad de complexona III 0,1 M, que se ha empleado en la valoración del CaO. Más tarde se incorpora una punta de espátula del indicador negro de eriocromo "T". Se valora hasta obtener un color azul-verde, que ya no se modifica al añadir más complexona.

Estequiométricamente $1 \mathrm{~mol}$ de complexona III corresponde a $1 \mathrm{~mol}$ de óxido magnésico, es decir, $1 \mathrm{ml}$ de complexona III 0,1 M corresponde a 4,038 $\mathrm{mg}$ de óxido de magnesio.

Los $\mathrm{ml}$ de solución de complexona empleados para la valoración del calcio y del magnesio, menos los ml empleados para el calcio, multiplicados por 2,016, proporcionan el contenido en $\%$ de magnesia en la muestra (con un peso de $1 \mathrm{~g}$ ).

\section{Reactivos empleados}

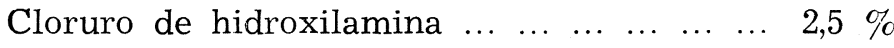

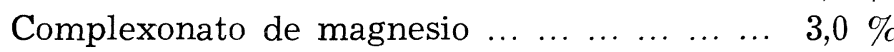

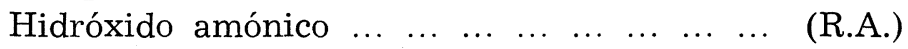

Solución de Complexona $0,1 \mathrm{M} \ldots \ldots \ldots \ldots \ldots$ para su preparación ver determinación del óxido de calcio.

Indicador de negro de eriocromo "T" ... ... ... (R.A.)

\section{La determinación del óxido magnésico en el ensayo de rutina de los cementos}

Para la T.F.B. suponía mucho tiempo la determinación gravimétrica del magnesio, realizada hasta ahora en el control de rutina de los cementos. El siguiente método proporciona un acortamiento considerable de tiempo. Del procedimiento resulta a la vez, sin mayor trabajo, el contenido en óxido cálcico del cemento.

\section{METODO DE TRABAJO}

\section{Determinación complexométrica del calcio y del magnesio en cementos}

Se toma $1 \mathrm{~g}$ de la muestra de cemento y se pone en un vaso de vidrio de $250 \mathrm{ml}$ (forma alta). Se añaden $10 \mathrm{ml}$ de anhídrido acético y $10 \mathrm{ml}$ de agua destilada. Se calienta el conjunto suavemente hasta que la reacción se completa (solución de color rosáceo).

A continuación se añade lentamente agua regia hasta que la solución se torna amarillo claro. Se añaden $20 \mathrm{ml}$ de agua destilada y se calienta de 5 a 10 minutos, una vez más, no con demasiada intensidad.

El conjunto se hace pasar por el filtro (banda blanca $11 \mathrm{~cm} \varnothing$ ). El residuo se lava dos veces con ácido clorhídrico al $5 \%$ y veinte veces con agua caliente destilada. El producto filtrado se pone en un matraz aforado de $500 \mathrm{ml}$ y se completa con agua destilada.

\section{Determinación del calcio}

Se toman:

- $100 \mathrm{ml}$ de la solución,

- $200 \mathrm{ml}$ de agua destilada,

- $20 \mathrm{ml}$ de trietanolamina al $50 \%$,

- $3 \mathrm{~g}$, aproximadamente, de hidróxido de sodio en lentejas (R.A.).

Se añaden inmediatamente $20 \mathrm{ml}$ de complexona $0,1 \mathrm{M}$. Todos los cementos portland suizos necesitan más de $20 \mathrm{ml}$ de complexona $0,1 \mathrm{M}$.

A continuación se añade una punta de espátula de indicador HHSNN puro. Entonces se continúa la valoración hasta que la solución se torna de un color azul puro. 
COMPARACION DE ANALISIS GRAVIMETRICO Y COMPLEXOMETRICO

\begin{tabular}{|c|c|c|c|c|c|c|c|c|c|c|c|c|c|c|c|c|}
\hline $\begin{array}{c}\text { Entrada } \\
\text { o } \\
\text { llegada }\end{array}$ & Marca & K. n.o & Calidad & $\begin{array}{c}\mathrm{SiO}_{2} \mathbf{G} \\
(\%)\end{array}$ & $\begin{array}{c}\mathbf{S i O}_{2} \mathbf{K} \\
(\%)\end{array}$ & $\hat{(\%)}$ & $\begin{array}{c}\mathbf{A l}_{2} \mathbf{O}_{3} \quad \mathbf{G} \\
(\%)\end{array}$ & $\begin{array}{c}\mathbf{A l}_{2} \mathbf{O}_{3} \mathbf{K} \\
(\%)\end{array}$ & $\underset{(\%)}{\Delta \mathbf{d}}$ & $\begin{array}{r}\mathbf{F e}_{2} \mathbf{O}_{3} \\
(\%)\end{array}$ & $\underset{(\%)}{\mathbf{C a O}} \mathbf{G}$ & $\underset{(\%)}{\mathbf{C a O} \mathbf{K}}$ & $\underset{(\%)}{\Delta d}$ & $\underset{(\%)}{\operatorname{Mgo} G}$ & $\underset{(\%)}{\operatorname{MgO}} \mathbf{K}$ & $\underset{(\%)}{\Delta \mathbf{d}^{\mathbf{d}}}$ \\
\hline $10-1-63$ & & 4672 & $\mathrm{PCS}_{5}$ & 2141 & 21.41 & 0.00 & 5.61 & 5.70 & +0.09 & 2.35 & 62.84 & 62.81 & -0.03 & 1.85 & 1.85 & 0.00 \\
\hline $11-1-63$ & & 4675 & $\mathrm{PCS}_{5}$ & 21.51 & 21.49 & -0.02 & 4.69 & $460^{\circ}$ & -0.09 & 2.90 & 6402 & 64.07 & +0.05 & 1.65 & 1.52 & -0.13 \\
\hline $12-1-63$ & & 4676 & $\mathrm{PCS}_{5}$ & 21.50 & 21.45 & -0.05 & 6.11 & 6.10 & -0.01 & 2.44 & 62.61 & 62.53 & -0.08 & 2.33 & 2.32 & -0.01 \\
\hline $11-1-63$ & & 4677 & $\mathrm{PCS}_{5}$ & 21.49 & 21.64 & +0.15 & 5.71 & 5.51 & -0.20 & 2.64 & 63.17 & 63.09 & -0.08 & 0.93 & 0.90 & -0.03 \\
\hline $17-1-64$ & & 4882 & $\mathrm{PC}$ & 21.21 & 21.20 & -0.01 & 4.08 & 4.04 & -0.04 & 3.02 & 64.28 & 64.21 & -0.07 & 1.36 & 1.21 & -0.15 \\
\hline $20-1-64$ & & 4884 & $\mathrm{PC}$ & 21.27 & 21.12 & -0.15 & 5.40 & 5.49 & +0.09 & 2.39 & 60.94 & 60.79 & -0.15 & 2.42 & 2.37 & -0.05 \\
\hline $17-1-64$ & & 4879 & $\mathrm{PCS}_{5}$ & 22.98 & 23.09 & +0.11 & 4.32 & 4.27 & -0.05 & 2.32 & 63.39 & 63.23 & -0.16 & 1.93 & 1.71 & -0.22 \\
\hline $10-1-64$ & & 4881 & $\mathrm{PCS}_{5}$ & 21.72 & 21.65 & -0.07 & 6.86 & 6.59 & -0.27 & 2.48 & 61.70 & 61.85 & +0.15 & 1.50 & 1.23 & -0.27 \\
\hline $20-1-64$ & & 4886 & $\mathrm{PCS}_{5}$ & 20.83 & 20.95 & +0.12 & 5.72 & 5.51 & -0.21 & 3.38 & 62.88 & 62.95 & +0.07 & 1.30 & 1.11 & -0.19 \\
\hline $21-1-64$ & & 4888 & $\mathrm{PCS}_{5}$ & 19.86 & 19.87 & +0.01 & 4.94 & 4.79 & -0.15 & 1.95 & 62.43 & 62.25 & -0.18 & 1.79 & 1.61 & -0.18 \\
\hline $22-1-64$ & & 4891 & $\mathrm{PCS}_{5}$ & 22.04 & 22.08 & +0.04 & 4.15 & 4.06 & -0.09 & 2.41 & 63.21 & 63.23 & +0.03 & 3.07 & 2.82 & -0.25 \\
\hline $7-11-63$ & & 4865 & Ferrari & 23.59 & 23.61 & +0.02 & 1.89 & 1.90 & +0.01 & 5.68 & 64.49 & 64.49 & 0.00 & 1.53 & 1.41 & -0.12 \\
\hline $10-5-63$ & & 4740 & Ferrari & 21.73 & 21.56 & -0.17 & 3.33 & 3.25 & -0.08 & 6.61 & 62.15 & 62.24 & +0.09 & 1.53 & 1.43 & -0.10 \\
\hline $10-5-63$ & & 4741 & Ferrari & 21.26 & 21.22 & -0.04 & 3.45 & 3.46 & +0.01 & 6.43 & 62.29 & 62.38 & +0.09 & 1.61 & 1.51 & -0.10 \\
\hline $1-4-64$ & & 4920 & Ferrari & 19.54 & 19.68 & +0.14 & 3.66 & 3.70 & +0.04 & 6.95 & 62.86 & 62.95 & +0.09 & 1.79 & 1.61 & -0.18 \\
\hline \multicolumn{6}{|c|}{ Desviación media: } & $0.073 \%$ & & & $0.094 \%$ & & & & $0.088 \%$ & & & $0.132 \%$ \\
\hline \multicolumn{6}{|c|}{ Desviación máxima: } & $+0.15 \%$ & & & $+0.09 \%$ & & & & $+0.15 \%$ & & & $0.00 \%$ \\
\hline \multicolumn{6}{|c|}{ Desviación mínima: } & $-0.17 \%$ & & & $-0.27 \%$ & & & & $-0.18 \%$ & & & $-0.27 \%$ \\
\hline \multicolumn{6}{|c|}{ Dispersión $\mathrm{S}$ : } & 0.061 & & & 0.079 & & & & 0.046 & & & -0.085 \\
\hline \multicolumn{6}{|c|}{ Coeficiente de variación (relativo): } & $0.456 \%$ & & & $1.970 \%$ & & & & $0.167 \%$ & & & $5.480 \%$ \\
\hline \multicolumn{6}{|c|}{ Número de cementos: 15} & & & & & & & & & & & \\
\hline
\end{tabular}




\section{Determinación del magnesio}

$\begin{array}{ll}\text { Se toman: } & \text { - } 100 \mathrm{ml} \text { de solución, } \\ & \text { - } 150 \mathrm{ml} \text { de agua destilada, } \\ & \text { - } 20 \mathrm{ml} \text { de cloruro amónico al } 30 \%, \\ & \text { - } 20 \mathrm{ml} \text { de cloruro de hidroxilamina al } 2,5 \%, \\ & \text { - } 3 \mathrm{ml} \text { de complexonato de magnesio (R.A.) al } 3 \% \text {, } \\ & \text { - } 10 \mathrm{ml} \text { de cianuro potásico (R.A.) al } 15 \%, \\ & 30 \mathrm{ml} \text { de hidróxido amónico (R.A.). }\end{array}$

Se añade la cantidad de complexona III $0,1 \mathrm{M}$ que se ha empleado en la valoración del $\mathrm{CaO}$. Ahora se añade una punta de espátula de indicador Erio "T". Se continúa la valoración hasta que se obtiene un color azul-verde.

\section{Aparatos}

Los aparatos son una probeta automática de precisión normal, de $50 \mathrm{ml}$ de capacidad. El vaso de vidrio se coloca sobre una base transparente, iluminada desde abajo con una lámpara para luz diurna tipo Sol-OSRAM de 200-230 V y $40 \mathrm{~W}$.

\section{Tolerancia de los resultados}

Conforme a las normas de la American Society for Testing and Materials, designación C 114-61 tenemos las siguientes tolerancias permitidas en el cuadro. La confrontación de este cuadro con el que figura anteriormente con el título "Comparación de análisis gravimétrico y complexométrico" muestra que en quince análisis comparativos las diferencias fueron:

\begin{tabular}{|ll|c|c|}
\hline \multicolumn{1}{|c|}{ Componente } & Fórmulas & $\begin{array}{c}\text { Entre dos } \\
\text { resultados }\end{array}$ & $\begin{array}{c}\text { Entre valores } \\
\text { extremos de tres } \\
\text { resultados }\end{array}$ \\
\hline Anhídrido silícico & $\mathrm{SiO}_{2}$ & 0,16 & 0,24 \\
Oxido de aluminio & $\mathrm{Al}_{2} \mathrm{O}_{3}$ & 0,20 & 0,30 \\
Oxido de hierro & $\mathrm{Fe}_{2} \mathrm{O}_{3}$ & 0,10 & 0,15 \\
Oxido cálcico & $\mathrm{CaO}$ & 0,20 & 0,30 \\
Oxido cálcico libre & $\mathrm{CaO}$ & 0,20 & 0,30 \\
Trióxido de azufre & $\mathrm{SO}_{3}$ & 0,16 & 0,15 \\
Oxido de magnesio & $\mathrm{MgO}$ & 0,16 & 0,24 \\
Pérdida al fuego & & 0,10 & 0,15 \\
Residuo insoluble & & 0,10 & 0,15 \\
Oxido sódico & $\mathrm{Na}_{2} \mathrm{O}$ & 0,03 & 0,05 \\
Oxido potásico & $\mathrm{K}_{2} \mathrm{O}$ & 0,03 & 0,05 \\
\hline
\end{tabular}

- en el caso del $\mathrm{SiO}_{2} \ldots \ldots \ldots . \quad \ldots \quad \ldots$ vez, $\quad$ - en el caso del $\mathrm{CaO}$ total $\ldots \ldots \ldots$ veces, - en el caso del $\mathrm{Al}_{2} \mathrm{O}_{3} \ldots \ldots \ldots .2$ veces, $\ldots$ en el caso del $\mathrm{MgO} \ldots \ldots \ldots 6$ veces, mayores que las tolerancias superiores entre dos resultados. En este caso, no se tuvo en cuenta que aquí se han comparado dos métodos de análisis diferentes.

\section{B I B L I O G R A F I A}

1. Borlera, M.: “Analisi rapida del cemento Portland e delle scorie di alto forno per via complessometrica”. L'Industria Italiana del Cemento, 1962.

2. Becker, F.: "Le dosage compléxométrique du fer, du calcium et du magnésium dans les ciments et les substances analogues". Revue des Matériaux de construction, París, n. ${ }^{\circ}$ 431-432, (1951).

3. Schwarzenbach G.: "Die komplexometrische Titration". Ferdinand Enke Verlag, Stuttgart (1960).

4. BuzzI, I. : "Contributo alla analisi rapida in Cementeria". L'Industria Italiana del Cemento, Febbraio, 1961.

5. Curtmann, L.: “Análisis químico cualitativo”. Manuel Marín, Editores. Barcelona, (1958).

6. Cowes Y Coleman.: "Análisis químico cuantitativo". Salvat. Editores, Barcelona, (1946).

7. Treadwell.: "Tratado de química analítica". Tomo II. Manuel Marín, Editores, Barcelona, (1949).

8. GuYe, F.: "Méthode d'analyse pour le clinker et le ciment Portland". Revue des Matériaux de construction, Paris, n. 437 Février 1952.

9. Burglen, L. et Longuet.: "Méthode nouvelle d'analyse chimique rapide des ciments". Revue des Matériaux de construction, Paris, n. 474 , Mars 1955.

10. BABOR, J. y IbARz, J.: “Química general moderna”. Manuel Marín, Editores, Barcelona 1963.

11. Cement and Concrete Association.: Technical Memorandum. April 1960.

12. Calleja, J., Fernandez J. y Triviño, F.: "Método complexométrico para el análisis rápido del cemento Portland". Instituto Eduardo Torroja. Madrid 1963.

13. AMERICAN SOciety For Testing AND Materials.: Standards part 4 (1961).

14. JANDER, G. und JAHR, K.: "Análisis volumétrico". Manuales tecnicos Labor. Labor Editorial, Barcelona 1944.

15. Bartholoma, Hans.: "Beitrag zur fällungsfreien Komplexometrischen Titration von Zementen", Tonindustrie-Zeitung Goslar, Mai 1962.

16. PrIBIL, R.: "Komplexometrie" VEB. Deutscher Verlag für Grundstoffindustrie, Leipzig 1960.

17. Stiglitz, P. et Cornet, J.: "Nouvelle méthode d'analyse des produits de cimenteries". Revue des Matériaux de construction n. 576. 